\title{
PREPARATION OF MOLECULARLY IMPRINTED POLYMERS FOR DIPHENYLAMINE REMOVAL FROM ORGANIC GUNSHOT RESIDUES
}

\author{
EDUARDO PEREIRA ${ }^{2 *}$, CÉSAR CÁCERES ${ }^{1}$, FABIOLA RIVERA ${ }^{1}$, BERNABÉ RIVAS ${ }^{2}$ PEDRO SÁEZ \\ ${ }^{1}$ Analytical Chemistry and Inorganic Department, ${ }^{2}$ Polymer Department \\ Faculty of Chemical Science, University of Concepción, Concepción, Chile \\ ${ }^{3}$ Sección Química y Física, Laboratorio de Criminalística Central, Policía de Investigaciones de Chile, PDI
}

\begin{abstract}
In order to obtain a better tool for collecting forensic evidence at the crime scene and the subsequent analysis of gunshot residue in laboratories, we propose the development of Molecularly Imprinted Polymers (MIPs) for the retention of diphenylamine (DPA), an organic compound coming from gunshot residues. This technique aims to achieve molecular recognition of a particular organic molecule, called target molecule. Once occurred recognition, this organic molecule may be removed, separated, and preconcentrated for further analysis.

For this, a series of MIPs and their analogs Non Molecularly Imprinted Polymers (NIPs) following a non-covalent strategy was synthesized. This was carried out by solution radical polymerization, using 1-vinyl-2-pyrrolidone (VP) as monomer, and diphenylamine as imprinting and target molecule. The retention capacity of MIPs was studied by HPLC and UV-VIS spectroscopy, meanwhile adsorption mechanism was studied by isothermal and kinetic assays. Resin yields ranged from $25 \%$ to $100 \%$. The results show that the variable that has more influence on DPA retention is the amount of crosslinking, wherein a greater amount of crosslinking results in higher retention of DPA, achieving values up to $90 \%$. There is a clear difference in adsorption capacity between MIPs and NIPs, where MIPs show higher adsorption capacity reaching a double or triple retention value. Kinetic studies show that it is achieved a high retention capacity within 5 min of contact. The results show that the fundamental mechanism for molecular recognition displayed by the imprinting effect can be attributed at two processes: the pre-organization of complementary functional groups present in the polymer and the template and selective cavity formation which complement the template size. Binding factors and selectivity combined effects contributing to molecular recognition of diphenylamine (DPA) by MIPs.
\end{abstract}

\section{INTRODUCTION}

The smokeless powder is the main driver of civilian and military ammunition and explosives and is the most common low intensity used to make improvised explosive devices such as pipe bombs. This type of smokeless powder can contain up to 23 organic compounds [1] is mainly produced by the thrusters of nitrocellulose (NC). There are also other additives key energetic material including nitroglycerine (NG) and stabilizers of diphenylamine (DPA), $\mathrm{N}$-nitrosodiphenylamine (NnDPA) and ethyl centralite (N, N'-diethyl-N, N'diphenylurea) (EC) [2].

Determination of the additive has important forensic applications. In the investigation of improvised explosive devices using smokeless powder, after the explosion residues are collected for laboratory evaluation of additives in half characteristic of the powders collected for subsequent determination of the source $[1,2]$.

Analysis of organic gunshot residues (OGSR) resulting from incomplete combustion of smokeless powder, emerges as an alternative approach for the detection of firearms or explosives [3-5]. The gun detection depends on the measurement characteristic of the organic components of the powder. The most common are the propellant, nitroglycerin (NG), and stabilizers including ethyl centralite (EC) and diphenylamine (DPA).

Organic components of gunpowder are not found in the general population, due to this fact, the detection of these additives on clothing or hands of a suspect is a good indicator that he has handled or fired a gun [6]. Generally, a tape to collect samples of organic waste firing is used [7-10]. However, the use of adhesives may lead to interference in the extraction and measurement of characteristic components of the OGSR. The adhesive polymer dissolved in a solvent used for the recovery of OGSR of the smokeless powders, is limited in the analysis, including methods based on aqueous extract by precipitation and separation of the interference spectrum of UV absorption of the organic compound adhesive $[11,12]$.

Cleaning and sample preconcentration is usually necessary to achieve lower detection limits in analyzing OGSR. Solid Phase Extraction (SPE) using selective reusable materials can be an excellent alternative for the detection of these substances. In this context, polymer resins have gained attention as novel type of sorbent with attractive properties. Particularly, a special class of polymer called "Molecularly Imprinted Polymers (MIPs)" arise with tremendous potential in this kind of applications [13-15].

Molecular imprinting is a way of creating recognition sites in polymeric materials. In fact, molecular imprinting of synthetic polymers is a process where functional and cross-linking monomers are polymerized or copolymerized in the presence of an imprint molecule, which acts as a molecular template. The functional monomers initially form a complex with the imprint molecule, and following polymerization, their functional groups are held in position by the highly cross-linked polymeric backbone. Subsequent removal of the imprint molecule reveals binding sites that are complementary in size and shape. In that way, a molecular memory is introduced into the polymer, which is now capable of selectively rebinding the target molecule [16-22].

Molecularly Imprinted Polymers have been used in several analytical techniques, including liquid chromatography, capillary electrochromatography, solid phase extraction, ligand binding assay, and sensor technology. Analytical separation field most close to practical realization is probably that of SPE. Several groups have already applied MIP-based on SPE to biological and environmental samples and this technique may well be accepted generally in the not-to-distant future. The technique has variously been referred to as MIP-SPE or MISPE. Following the first study on MIP-SPE employing a MIP selective for pentamidine in a model on-line sample enrichment of a spiked urine sample $[23,24]$ a number of groups have presented SPE applications quantitative extraction of the sample and elution of for various types of analytes.

In this work was performed the synthesis of a MIPs and NIPs based on 1-vinyl-2-pyrrolidone monomer, using DPA as imprinting and target molecule. The synthesis was conducted following an experimental design in order to optimize the variables that affect the process of retention of DPA in order to assessed the maximum adsorption capacity in batch method.

\section{EXPERIMENTAL SECTION}

2.1 Materials.

All the reagents and solvents were of analytical grade or higher and were used without further purification. The reagents: 1,4-divinylbenzene (DVB), $80 \%$, 1-vinyl-2-pyrrolidone (VP), $\geq 99 \%, 2,2^{\prime}$-azobisisobutyronitrile (AIBN) (SIGMA ALDRICH), absolute ethanol (ETOH) grade P.A., absolute ethanol grade HPLC, Diphenylamine (DPA) 98\% (MERCK).

2.2 Synthesis of molecularly imprinted polymers (MIPs) and non imprinted polymers (NIPs).

The synthesis of MIPs were carried out following an experimental design developed in the MODDE 7.0 software. The experimental design generate a total of 19 synthesis (see table No 1) varying the amount of crosslinker, imprinting molecule, solvent, and initiator reagents. The amount of monomer, $2.0 \mathrm{~g}$ was maintained constant in all the experiments.

The required amount of monomer $(2.0 \mathrm{~g} \mathrm{VP})$ is weighed and brought in a polymerization flask of $50 \mathrm{~mL}$, then added the solvent (ETOH) and the amount of imprinting molecule (DPA). The mixture was stirred for 5 min to obtain a homogeneous solution, after stirring was allowed to stand for $30 \mathrm{~min}$. After, 
the required volume of crosslinker reagent (DVB) was added and stirred for 5 min. Following, the required amount of initiator (AIBN) was dissolved in the minimum amount of solvent. This mixture was bubbled with $\mathrm{N}_{2}(\mathrm{~g})$ with a Pasteur pipette for $5 \mathrm{~min}$ and then fill the tube with a balloon inflated with $\mathrm{N}_{2}(\mathrm{~g})$ for generating an inert atmosphere. The tubes were brought to a thermostatic bath at $70^{\circ} \mathrm{C}$ for $24 \mathrm{~h}$. Once the synthesis is complete the MIPs were removed from the polymerization flask and carried out a crucible coupled to a vacuum filtration system, and then proceed to wash the polymer with $300 \mathrm{~mL}$ of ethanol and $200 \mathrm{~mL}$ of milliQ water. After washing the resin, this is put into a Petri dish in an oven at $40^{\circ} \mathrm{C}$, up to constant weight.

Table 1. Experimental conditions for the synthesis of MIP according to experimental design

\begin{tabular}{|c|c|c|c|c|}
\hline $\begin{array}{c}\text { No of } \\
\text { Exp. }\end{array}$ & $\begin{array}{c}\text { Mass of } \\
\text { Initiator } \\
\text { AIBN (g) }\end{array}$ & $\begin{array}{c}\text { Volume of } \\
\text { Crosslinker } \\
\text { DVB (mL) }\end{array}$ & $\begin{array}{c}\text { Volume } \\
\text { of Solvent } \\
\text { Ethanol }(\mathrm{mL})\end{array}$ & $\begin{array}{c}\text { Mass of } \\
\text { Imprinting } \\
\text { Molecule } \\
\text { DPA (g) }\end{array}$ \\
\hline MIP-1 & 0.0296 & 1.30 & 2 & 0.3050 \\
\hline MIP-2 & 0.0886 & 1.30 & 2 & 0.3050 \\
\hline MIP-3 & 0.0296 & 7.81 & 2 & 0.3050 \\
\hline MIP-4 & 0.0886 & 7.81 & 2 & 0.3050 \\
\hline MIP-5 & 0.0296 & 1.30 & 10 & 0.3050 \\
\hline MIP-6 & 0.0886 & 1.30 & 10 & 0.3050 \\
\hline MIP-7 & 0.0296 & 7.81 & 10 & 0.3050 \\
\hline MIP-8 & 0.0886 & 7.81 & 10 & 0.3050 \\
\hline MIP-9 & 0.0296 & 1.30 & 2 & 0.9130 \\
\hline MIP-10 & 0.0886 & 1.30 & 2 & 0.9130 \\
\hline MIP-11 & 0.0296 & 7.81 & 2 & 0.9130 \\
\hline MIP-12 & 0.0886 & 7.81 & 2 & 0.9130 \\
\hline MIP-13 & 0.0296 & 1.30 & 10 & 0.9130 \\
\hline MIP-14 & 0.0886 & 1.30 & 10 & 0.9130 \\
\hline MIP-15 & 0.0296 & 7.81 & 10 & 0.9130 \\
\hline MIP-16 & 0.0886 & 7.81 & 10 & 0.9130 \\
\hline MIP-17 & 0.0591 & 4.56 & 6 & 0.6090 \\
\hline MIP-18 & 0.0591 & 4.56 & 6 & 0.6090 \\
\hline MIP-19 & 0.0591 & 4.56 & 6 & 0.6090 \\
\hline
\end{tabular}

The non molecularly imprinted polymers (NIPs) were synthesized under the same conditions as MIPs but in the absence of the imprinting molecule.

2.3 Removal of the imprinting molecule.

The removal of the imprinting molecule (DPA) was performed with the dry MIPs. These are brought into a beaker of $250 \mathrm{~mL}$, then was added 150 $\mathrm{mL}$ of methanol, stirred for a few min and allowed to stand for $1 \mathrm{~h}$. Finally, the supernatant was removed through decantation. The procedure was repeated 3 times. After that the resin was brought to a crucible coupled to a vacuum filtration system, filtered and brought to a Petri dish to subsequently carry the resin to the oven at $40{ }^{\circ} \mathrm{C}$ up to constant weight.

\subsection{Grinding and sieving of MIPS and NIPS.}

Once dried, MIPs were sieved using sieves proceeded particle size of 250 $\mathrm{mm}, 180 \mathrm{~mm}$ and $100 \mathrm{~mm}$. For all subsequent studies the used fraction of particle size was $100-180 \mathrm{~mm}$. The resin remaining in the fraction above 180 $\mathrm{mm}$ was taken to a mill to obtain the desired granulometry of the resin.

2.6 Characterization of MIPS and NIPs through FT-IR and SEM

The infrared spectra of the MIPs samples in KBr pellets were obtained on a Nicolet Magna 550, in the range of 4000-400 $\mathrm{cm}^{-1}$. Moreover the samples were analyzed by scanning electron microscopy (SEM) in a JEOL JSM-6380 $\mathrm{LV}$ using an acceleration voltage of $20 \mathrm{kV}$ and different magnification ranges. 2.5 Retention studies for MIPs and NIPs in batch method.

In a $15 \mathrm{~mL}$ falcon tube was added $100 \mathrm{mg}$ of MIP or NIP and $10 \mathrm{~mL}$ of $100 \mathrm{ppm}$ standard solution of diphenylamine. This mixture was stirred for 1 $\mathrm{h}$ on a reciprocal shaker. Then centrifuged for $5 \mathrm{~min}$ and the supernatant was filtered through PVDF membrane filter $0.22 \mathrm{~mm}$ and placed into an Eppendorf tubes. The concentration of DPA in the supernatant was measured by HPLC or UV-VIS spectrometry.

The HPLC method was developed using a YL 9100 HPLC System with a quaternary pump YL9110, Degasser YL9101, UV/VIS diode array Detector YL 9160, Injector, loop of $20 \mu \mathrm{L}$, integration system and data logging ClarityChromatography SW, C8 Symmetry ${ }^{\circledR}$ column, $5 \mu \mathrm{m}, 4.6 \times 250 \mathrm{~mm}$ Waters, membrane filter PVDF $0.45 \mu \mathrm{m}, 47 \mathrm{~mm}$ of Diameter, Durapore $\mathbb{R}$, and a syringe of $50 \mu \mathrm{L}$ SGE Analytical Science. The chromatographic conditions of analysis of DPA were; mobile phase: $\mathrm{MeOH}: \mathrm{H}_{2} \mathrm{O}$ 80:20, column temperature: $25^{\circ} \mathrm{C}$, flow of mobile phase: $0.7 \mathrm{~mL} / \mathrm{min}$, time of Analysis: $15 \mathrm{~min}$, injection volume: $20 \mu \mathrm{L}$, wave length: $284 \mathrm{~nm}$.

\subsection{Kinetics studies.}

In order to measure the retention capacity at different contact times, 100 $\mathrm{mg}$ of MIP or NIP and $10 \mathrm{~mL}$ of $100 \mathrm{ppm}$ standard solution of DPA were added to each one of -8 falcon tubes. The 8 tubes were brought to a reciprocal shaker, removing the tubes at $0,5,10,15,30,45,60$ and $90 \mathrm{~min}$. After that, the tube was rushed to the centrifuge for $3 \mathrm{~min}$ and the supernatant liquid was filtered with PVDF membrane filter $0.22 \mathrm{~mm}$ and placed into an Eppendorf tube.

The concentration of DPA in the supernatant was measured by HPLC or UV-VIS spectrometry.

\subsection{Adsorption isotherms.}

The adsorption isotherms were performed with 8 different concentrations of DPA in the range of 50 to $5000 \mathrm{mg} \mathrm{L}^{-1}$ and 4 different temperatures 25,30 , 35 , and $40^{\circ} \mathrm{C}$. To each falcon tube were added $50 \mathrm{mg}$ of MIP or NIP and 10 $\mathrm{mL}$ of the standard solution of DPA of the corresponding concentration. They were then taken to a shaking water bath for $24 \mathrm{~h}$ at a speed of $140 \mathrm{rpm}$ at the corresponding temperature. At the end, the tubes were removed and centrifuged for $2 \mathrm{~min}$ at $4000 \mathrm{rpm}$. The supernatant was brought to an Eppendorf tube and subsequently measuring the concentration of equilibrium DPA by HPLC or UV-VIS spectrometry.

\section{RESULTS AND DISCUSSION}

\subsection{Synthesis and Characterization of MIPs and NIPS}

According to the experimental design, a total of 19 MIPs produced in different experimental conditions were obtained. Figure 1 shows the yield for each one of them.

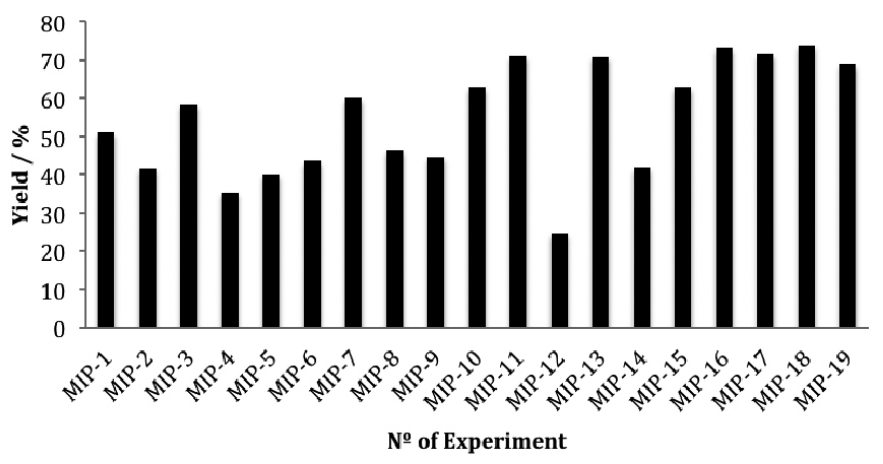

Figure 1. Yield of the synthesis of MIPs in different experimental conditions

The highest yields, between $70 \%$ and $80 \%$, were for the MIPs obtained in the experimental conditions No 17, No. 18, and No. 19. It should be noted that these MIPs were synthesized in the same experimental conditions (see table 1), indicating that the polymerization process is reproducible under the same synthesis conditions. These experimental conditions correspond to a mean value of the amount of initiator, crosslinker, solvent, and imprinting molecule. The lowest yield, $35.3 \%$ and $24.6 \%$ were for the MIPs obtained in the experimental conditions No. 4 and No 12 respectively. These experimental conditions are characterized for higher amount of initiator, crosslinker, and imprinting molecule.

Figure 2 shows the FT-IR spectrum obtained for MIP-13. In general, the FT-IR spectra for all the MIPs are very similar each to other, consistent with the fact that all the products were synthesized based on same monomer, 
1-vinyl-2-pyrrolidone and crosslinker, divinylbenzene. It is also important to note that there is no significant difference between MIPs and NIPs. Poly $\mathrm{N}$-vinylpyrrolidone has a characteristic, a relatively broad, infrared band at $1682 \mathrm{~cm}^{-1}$ that is attributed to a mixed mode containing major contributions from the carbonyl and N-C stretching vibrations. The absorption band at 2923 $\mathrm{cm}^{-1}$ corresponding to the asymmetrical vibration of $\mathrm{C}-\mathrm{H}$ bond of the $-\mathrm{CH}_{2}$ polymer chain and the broad band centered at $3493 \mathrm{~cm}^{-1}$ corresponds to water from humidity.

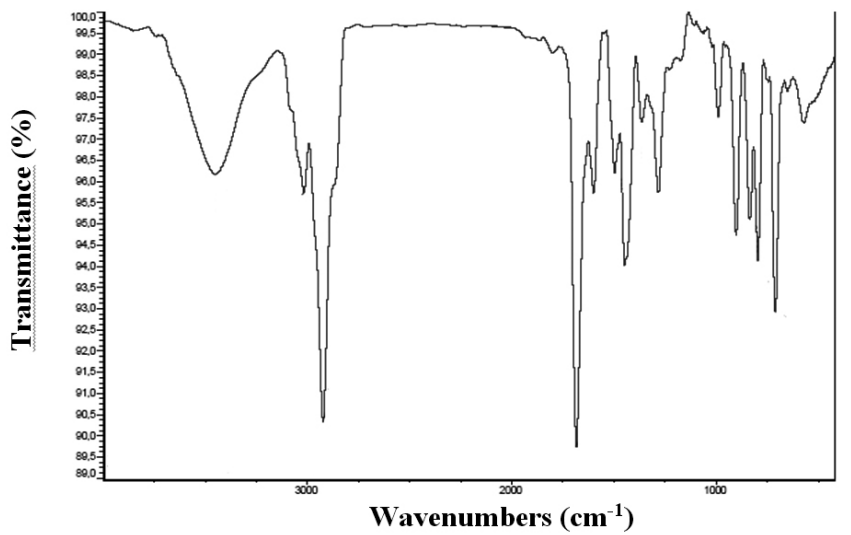

Figure 2. FT-IR spectrum for MIP-13 in $\mathrm{KBr}$.

Figures 3a, 3b, 3c and 3d show the SEM micrographs for MIP-13, MIP-17, NIP-13, and NIP-17 respectively. The micrographs reveal clusters of spherical and tight particle size distribution for all cases. For MIP-17 and NIP-17 adopts a more spherical shape and a larger particle size. Either way the above to date, the effect of the template on the final particle size has not been sufficiently studied. In general in the synthesis of MIPs and NIPs are tries obtaining particles defined size and shape. To this should be controlled polymerization method since this affects the external and internal morphology of the polymer. Generally in a solution polymerization the particles are irregular in shape and size due to milling and sieving process, even due to some interaction sites that can be destroyed. To obtain spherical particles have been used some types of polymerization such as suspension polymerization, precipitation polymerization, interfacial polymerization, among others. Despite the difficulty of obtaining spherical particles by the solution polymerization has been reported that the use of excess porogenic solvent contributes to the formation of such particles because the polymer chains in formation are not able to occupy the total volume solvent to produce a dispersion of spherical particles separate from the solution. Also, has been reported that a slow polymerization process could result in the formation of spherical particles as it gives more time for the formation of such particles.
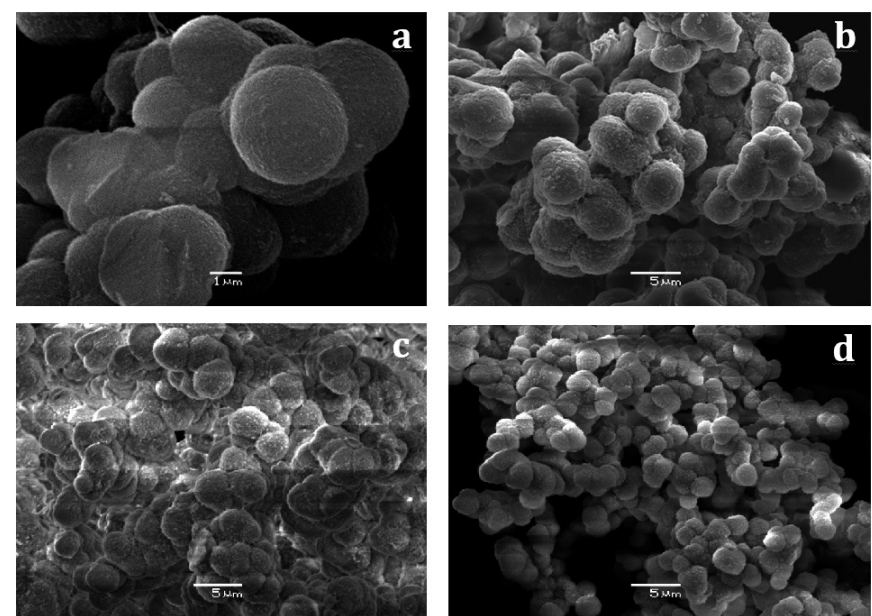

Figure 3. SEM images of (a) MIP-13. (b) MIP-17, (c) NIP-13, and (d) NIP-17
Another factor that can influence this fact is the use of a hydrophobic monomer and hydrophilic solvent, which may result in dissolution repulsion causing interfacial polymerization type, wherein this repulsion generated spherical particles. The last is the more plausible explanation for the morphology of our synthesized MIPs and NIPs.

\subsection{Diphenylamine retention capacity of the synthesized MIPs}

The fundamental mechanism for molecular recognition displayed by the imprinting effect can be attributed to two processes: the pre-organization of complementary functional groups present in the polymer and the template and selective cavity formation that complement the template size. Binding factors and selectivity combined effects contributing to molecular recognition of MIPs.

Figure 4 shows the retention capacity of DPA by the 19 MIPs obtained according to the experimental design. Most of the MIPs show a similar retention of DPA with lower values for MIP-4 and MIP-11, around 80\% and higher values for MIP-9, MIP-14, and MIP-17, around 90\%. These high retention capacity indicate a strong interaction between DPA and the basic

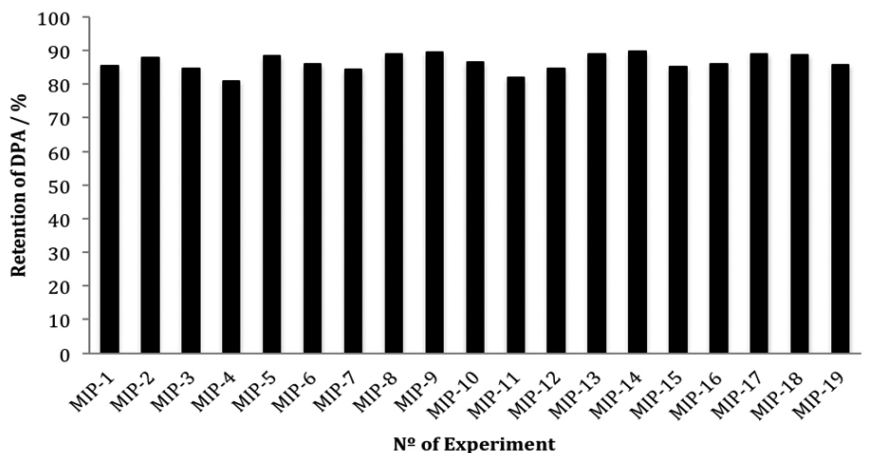

Figure 4. DPA retention capacity of MIPs synthesized according to the experimental design

repeat unit of $\mathrm{N}$-vinylpyrrolidone. The main interaction is the hydrogen bond between the amino group of the DPA and the carbonyl group of $\mathrm{N}$-vinylpyrrolidone unit (see figure 5). Also is possible see that the retention capacity is slightly affected by the experimental variables used.

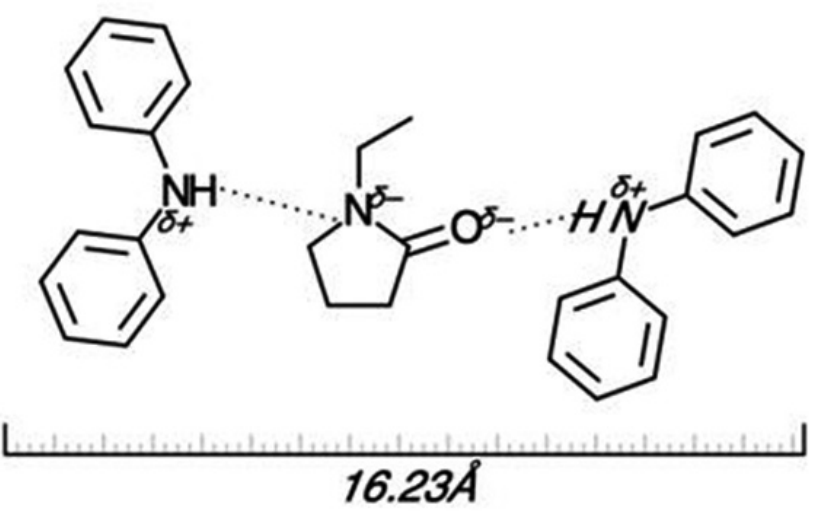

Figure 5. Scheme of possible nonbonding interactions between DPA and the monomer 1-vinyl-2-pyrrolidone

In order to determine the simultaneous influence of the crosslinker content in MIPs yield and DPA retention capacity, a ternary plot of theses three variables was obtained (see figure 6).

From these representations, different tendencies can be observed varying the amounts of crosslinker. Groups of three levels of crosslinker used, $25 \%, 50$ $\%$ and $75 \%$ are clearly observed. This indicates that the other studied variables, such as, amount of template molecule, solvent and initiator, have less effect on the yield and retention capacity of the MIPs obtained. However, considering the variable amount of crosslinker, it can be seen that this is the most important factor in the yield of the synthesis and at the same time is related, in a lesser 
extent, with retention capacity of MIPs. Thus, as the amount of crosslinking increases, the yield of the synthesis of MIPs decreases, with yields of about $25-30 \%$, while for lower amounts of crosslinker, the synthesis yield reaches higher values around of $80 \%$. In the synthesis systems with less amount crosslinking, decreases the likelihood of homopolymerization of this, so that more crosslinked MIPs are obtained, enhancing its yield and diminishing the loss in the different cycles of washing and drying.

With this in mind we proceed to take the best mini-MIPs, i.e. those with the best performance in the synthesis, and the best retention rates of DPA. The polymers were selected from the experiment No 13 and No 17. These particular experiments were synthesized again but in greater amount for subsequent experiments and were renowned as MIP I and MIP II. For these experiments the yield of the synthesis was $41.5 \%$ and $65.1 \%$ respectively. The analogous NIPs, used for template control, were synthesized in the same experimental conditions than MIPs but in absence of template molecule.

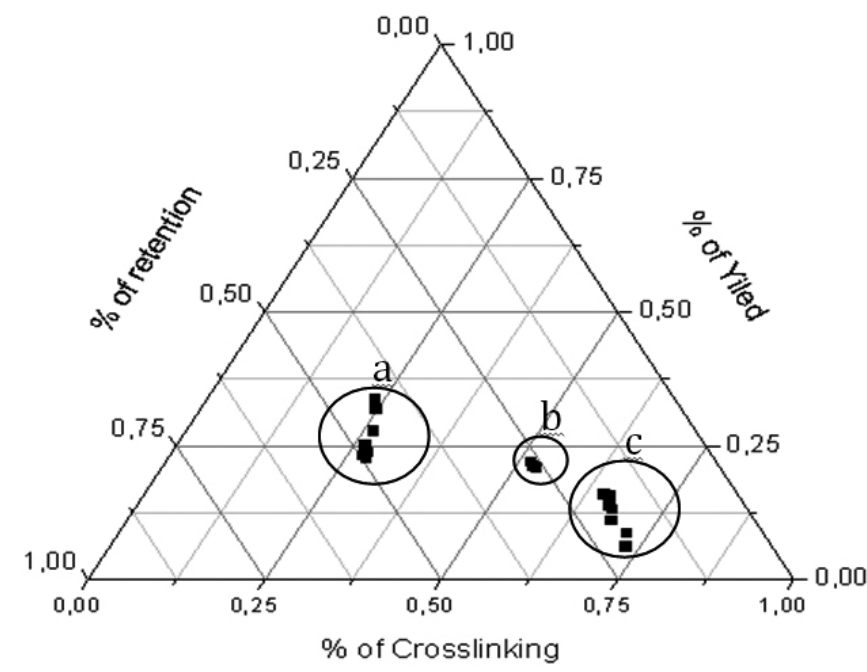

Figure 6. Ternary plot of DPA retention capacity for 19 MIPs. Crosslinker content (a) $50 \mathrm{~mol} \%$, (b) $175 \mathrm{~mol} \%$, and (c) $300 \mathrm{~mol} \%$

3.3 Diphenylamine retention studies by selected MIPs and NIPs.

As previously mentioned, the MIPs that showed higher performance and retention rate were synthesized in greater amounts. The retention capacity was studied in batch method by contact of MIP with a $100 \mathrm{ppm}$ DPA solution. After that, the DPA concentration in the supernatant was measured using UV-VIS spectroscopy. This was achieved with a DPA calibration curve using patterns from 2 to $10 \mathrm{ppm}$ (see figure 7).

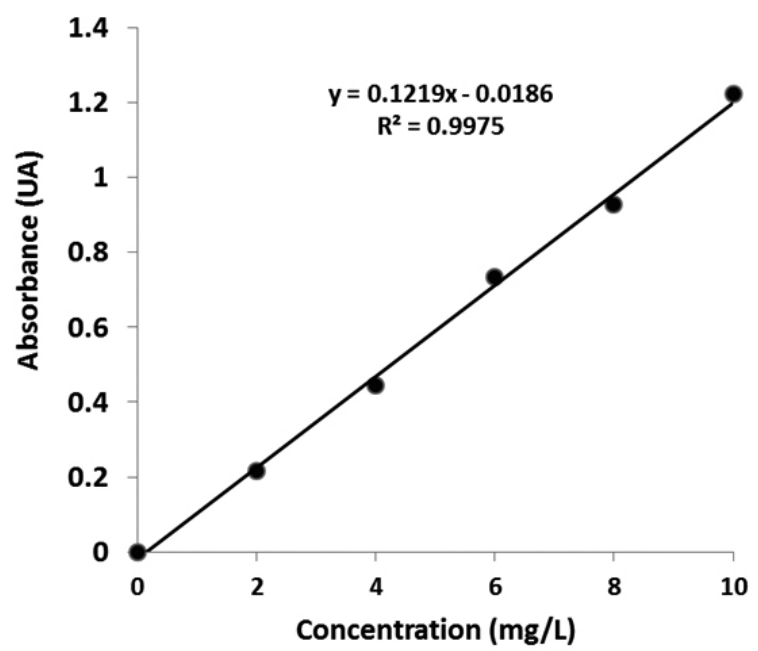

Figure 7. Calibration curve for DPA in UV-visible spectrophotometer, at 6 different concentrations, the solvent is 50-50 ethanol-water mixture.
In figure 8, DPA retention capacity for selected MIPs and NIPs are showed. The retention capacity for MIPs I and II are very similar, reaching values close to $75 \%$, meanwhile the retention capacity for NIP I and II are also similar between them and slightly lower than MIPs.

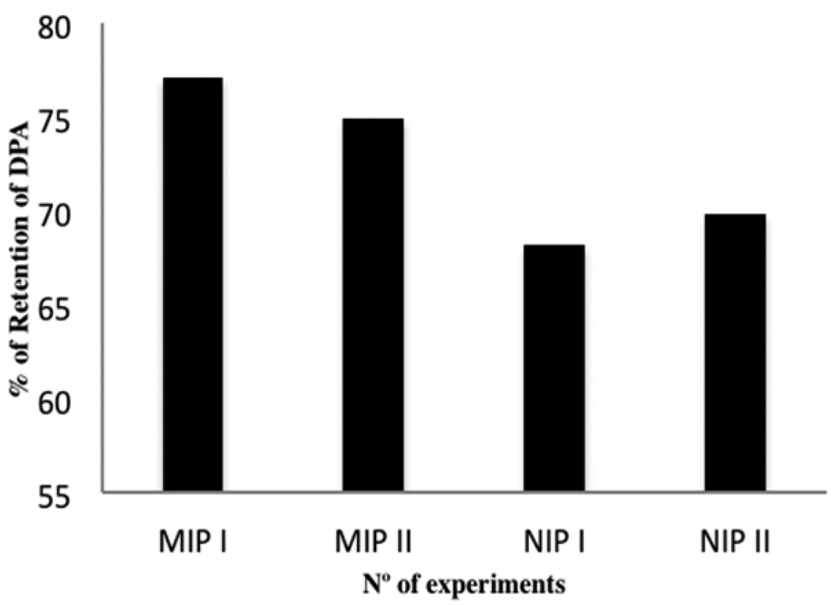

Figure 8. Retention of DPA for MIPs and NIPs

Because of the low increase in the retention capacity of MIPs compared with NIPs, we can not conclude on the formation of the cavities during the template process. In order to get clear conclusions on this subject we must perform a more detailed study of retention, this will be discussed later in the discussion of the adsorption isotherms.

\subsection{Adsorption isotherms.}

To determine the maximum adsorption capacity of polymer, adsorption isotherms are conducted to MIPs I and II and NIPs I and II. After obtaining the isotherms were fitted to two models of isotherms, Freundlich isotherms and Langmuir isotherms. With the data obtained from the isotherms were plotted linear expressions each isotherm model and then estimate the parameters associated with them.

\subsubsection{Freundlich and Langmuir linearized isotherms.}

Various mathematical models can be used to study experimental data adsorption isotherms. To analyze the data obtained in this work we use the Langmuir and Freundlich models. Fitting the data to the isotherm models can accurately describe the experimental results of the adsorption isotherms and to find the most appropriate model for our MIPs and NIPs.

From linearized Langmuir isotherm and Freundlich is possible to obtain the binding parameters, $\mathrm{m}$, a, maximum adsorption capacity (Qmax), among others. These parameters can be set which is resin, which also presents a greater retention, or surface type resins presented.

Table No 2 shows the parameters obtained for each isotherm model.

For this system, both models show excellent linearity $\left(\mathrm{R}^{2} \geq 97 \%\right)$ both MIPs to NIPs.

Comparing the correlation coefficients between the 2 isotherm models used we can see that there is a greater linearity in the Langmuir isotherm indicating that the data obtained from the isotherms are better fitted to this model.

As the parameter $\mathrm{m}$ obtained from the Freundlich isotherm indicates a homogeneous surface $(\mathrm{m}>1)$ with similar values between MIPs and NIPs.

Considering the Langmuir parameters we can conclude that the highest value for the maximum adsorption capacity corresponding to NIP I at $40^{\circ} \mathrm{C}$ whose value is $286 \mathrm{mg}$ of DPA/g of NIP. In general the values of the binding constant $(\mathrm{K})$ show an increase with increasing temperature. It is also important to note that in general for this system the values of adsorption capacity of MIPs are higher than in the NIPs in 2-fold maximum. 
Table 2. Binding parameters calculated from Langmuir and Freundlich isotherms linearized to MIPs and NIPs.

\begin{tabular}{|c|c|c|c|c|c|c|c|c|c|c|c|c|c|}
\hline \multirow[b]{3}{*}{ Sample } & \multirow[b]{3}{*}{$\begin{array}{c}\text { Temp } \\
\left({ }^{\circ} \mathrm{C}\right) \\
\end{array}$} & \multicolumn{6}{|c|}{ MIPs } & \multicolumn{6}{|c|}{ NIPs } \\
\hline & & \multicolumn{3}{|c|}{ Freundlich } & \multicolumn{3}{|c|}{ Langmuir } & \multicolumn{3}{|c|}{ Freundlich } & \multicolumn{3}{|c|}{ Langmuir } \\
\hline & & $\mathrm{a}$ & $\mathrm{m}$ & $\mathrm{R}^{2}$ & $\begin{array}{c}\text { Qmax } \\
(\mathrm{mg} / \mathrm{g})\end{array}$ & $\begin{array}{c}\mathrm{K}(\mathrm{L} / \\
\mathrm{mg})\end{array}$ & $\mathrm{R}^{2}$ & $\mathrm{a}$ & $\mathrm{m}$ & $\mathrm{R}^{2}$ & $\begin{array}{c}\text { Qmax } \\
(\mathrm{mg} / \mathrm{g})\end{array}$ & $\begin{array}{c}\mathrm{K}(\mathrm{L} / \\
\mathrm{mg})\end{array}$ & $\mathrm{R}^{2}$ \\
\hline I & 25 & 3013 & 1.426 & 0.981 & 139 & 360 & 1.000 & 4469 & 1.305 & 0.977 & 114 & 293 & 0.987 \\
\hline I & 30 & 8300 & 1.234 & 0.968 & 123 & 405 & 0.992 & 9687 & 1.182 & 0.977 & 123 & 405 & 0.992 \\
\hline I & 35 & 4874 & 1.358 & 0.995 & 108 & 465 & 0.994 & $*$ & $*$ & $*$ & $*$ & $*$ & $*$ \\
\hline I & 40 & 4787 & 1.342 & 0.989 & 99 & 505 & 0.986 & 5223 & 1.289 & 0.995 & 286 & 175 & 0.940 \\
\hline II & 25 & 3311 & 1.437 & 0.990 & 141 & 355 & 0.999 & 4047 & 1.344 & 0.995 & 156 & 213 & 0.998 \\
\hline II & 30 & 8078 & 1.239 & 0.959 & 156 & 320 & 0.999 & 7637 & 1.225 & 0.966 & 112 & 445 & 0.993 \\
\hline II & 35 & 4817 & 1.369 & 0.990 & 222 & 225 & 0.961 & 3036 & 1.454 & 0.980 & 94 & 530 & 0.984 \\
\hline II & 40 & 4722 & 1.359 & 0.989 & 161 & 310 & 0.999 & 3273 & 1.416 & 0.995 & 102 & 490 & 0.993 \\
\hline
\end{tabular}

For systems MIP I and MIP II are a clear trend, there is greater retention capacity in MIPs, comparing it with the NIPs. Also notice that the MIP II has a higher maximum retention capacity compared to system MIP I, this is because the MIP II system was synthesized using a higher percentage of cross-linking which could cause a more porous surface due a high crosslinking and hence greater retention.

a

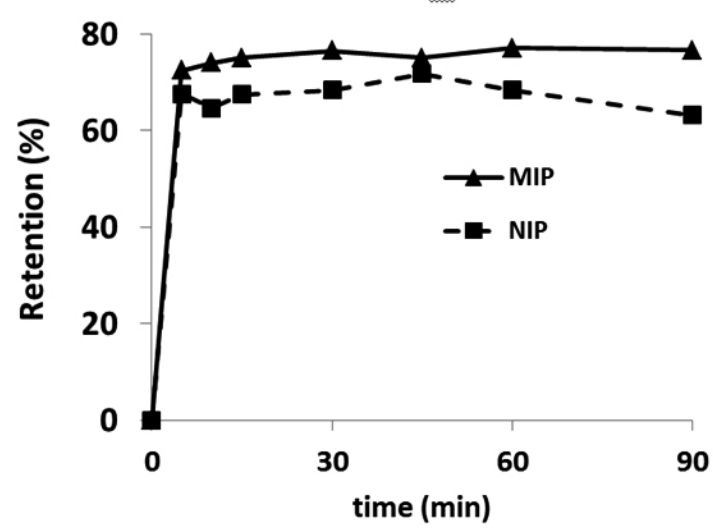

3.5 Kinetic study of the retention of DPA in MIPs.

DPA adsorption reached equilibrium phase within a very short time period as shown in Figure 9a and 9b, this is attributed to the utilization of available adsorption sites on the polymer surface is almost immediate. As this happens in a very short time, the cavities generated in the polymer are saturated provokes DPA that there is a considerable increase in the percentage of retention.

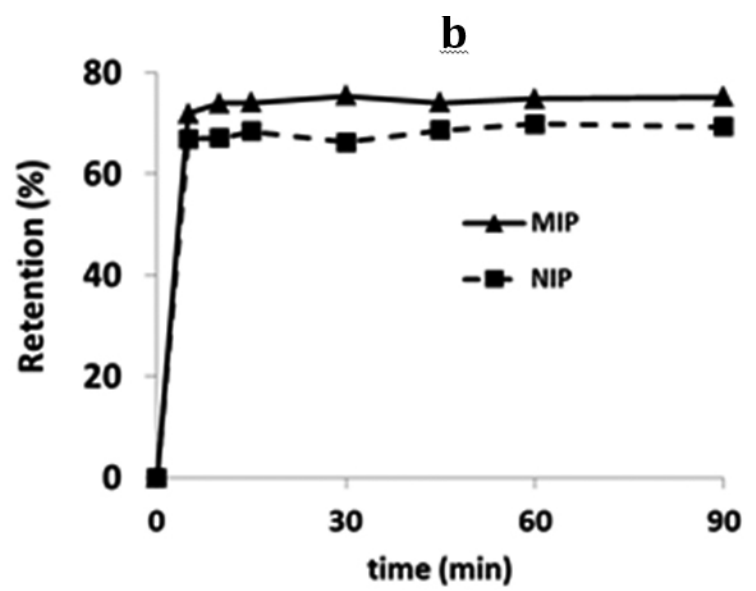

Figure 9. Kinetic study of MIPs and NIPs, study of retention capacity of MIPs and NIPs in function of Time; a) is for MIP I and NIP I and b) is for MIP II and NIP II

As for differences between MIPs and NIPs observed the same trend described above, similarly behaving MIP and NIP for a particular polymer. It is also important to note that the kinetic study shows the same tendency to retain detail in the study of MIPs and NIPs retention.

\section{CONCLUSIONS}

Using the solution radical polymerization it is possible to obtain polymers with good yields for most experiments. With this polymerization technique agglomerated spherical particles were obtained with regular and constant diameters close to 2.5 micrometers. The fact of obtaining spherical particles is very important because in spite of uncontrolled synthesis variables to obtain a particular particle type were obtained.

The use of multivariate analysis indicates that the variable most influential in the percentage yield of polymers and DPA retention is the amount of crosslinking.

The kinetic study shows that the retention of DPA is practically immediate, and showing a maximum retention rate in the first 5 min of contact

By comparing the maximum adsorption capacity for polymers is concluded that there is a difference between MIPs and NIPs, with the MIPs which have a higher retention capacity due to cavities generated during the printing process. This is an indication that such systems could be used in the near future to preconcentrate organic gunshot residues in order to help the police in solving the crime.

\section{ACKNOWLEDGEMENTS}

The authors thanks gratefully to research FONDECYT No 1100775 for the financial support. C. Cáceres thank to CONICYT for Doctoral Fellowship No 21110603. CIPA CONICYT REGIONAL/CIPA/R08C1002.

\section{REFERENCES}

1. C.M. Selavka, R.A. Strobel, R.E. Tontarski, The systematic identification of smokeless powders: an update, in: Proceedings of the Symposium on Analysis and Detection of Explosives.

2. C.L. Wallace, C.R. Midkiff Jr., in Smokeless powder characterization: an investigative tool in pipe bombings: J. Yinon (Ed.), Advances in Analysis 
and Detection of Explosives, Kluwer Academic Publishers, 1993; pp. 29-39.

3. S.J. Speers, K. Doolan, J. McQuillan, J.S. Wallace, J. Chromatogr. A 674 319 (1994).

4. T.G. Kee, D.M. Holmes, K. Doolan, J.A. Hamill, R.M.E. J. Forensic Sci. Soc. 30, 285 (1990).

5. Z. Wu, J. Yu, X. Zhang, C. Yang, C. Pan, X. Deng, Y. Wen, Z. Xu, J. Forensic Sci. 46, 495 (2001).

6. D.M. Northrop, Part 1, J. Forensic Sci. 46, 549 (2001).

7. D.M. Northrop, Part 2. J. Forensic Sci. 46, 560 (2001).

8. K. Bratin, P.T. Kissinger, R.C. Briner, C.S. Bruntlett, Anal. Chim. Acta 130, 295 (1981).

9. L. Leggett, P.F. Lott, Microchem. J. 39, 76, (1989).

10. D.M. Northrop, W.A. MacCrehan, J. Liq. Chromatogr. 15, 1041, (1992).

11. W.A. MacCrehan, M. Bedner, Forensic Sci. Int. 163, 119, (2006).

12. M.R. Reardon, W.A. MacCrehan, J. Forensic Sci. 46, 802, (2001).

13. M. Janotta, R. Weiss, B. Mizaikoff, O. Brüggemann, L. Ye, K. Mosbach, Int. J. Environ. Anal.Chem., 80, 1029, (2001).

14. L.I. Andersson, J. Chromatogr. B, 3, 745, (2000).
15. J.C. Bravo, R. M. Garcinuño, P. Fernández, J.S. Durand, Anal. Bioanal. Chem., 388, 1618, (2007).

16. R. Arshady, K. Mosbach, Makromol. Chem., 182, 687 (1981).

17. M.C. López,C. Pérez, M.D. García, J.L. Vilariño, M.G. Rodríguez, L.B. Losada Analytica Chimica Acta 721, 68 (2012).

18. X. Qi, X. Yang, B. Wang, H. Yang, A. Deng. Intern. J. Environ. Anal. Chem. 92, 268 (2012).

19. S. Sadeghi, A. Motaharian, A.Z. Moghaddam Sensors and Actuators B 168, $336(2012)$

20. A.C. Diasa, E.C. Figueiredo, V. Grassi, E.A Zagattob, M.A. Arrudaa, Talanta, 76, 988, (2008).

21. X. Song, J. Li, S. Xu, R.Y. Jiping Ma, C. Liao, D. Liu, J. Yu, L. Chen. Talanta 99, 75 (2012)

22. S. Ambrosini, S. Shinde, E. De Lorenzi, B. Sellergren. Analyst. 137, 249 (2012).

23. J. Olsen, P. Martin, I.D. Wilson, Anal. Commun. 35, 60, (1998).

24. Diasa A.C.B., Figueiredo E.C., Grassi V., Zagattob E.A.G., Arrudaa M.A.Z., Talanta, 76, 988, (2008) 